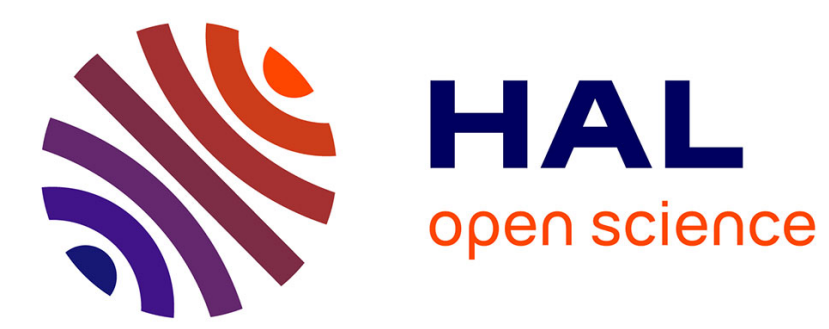

\title{
Soap Films Burst Like Flapping Flags
}

Henri Lhuissier, Emmanuel Villermaux

\section{To cite this version:}

Henri Lhuissier, Emmanuel Villermaux. Soap Films Burst Like Flapping Flags. Physical Review Letters, 2009, 103 (5), pp.054501. 10.1103/PhysRevLett.103.054501 . hal-00426813

\section{HAL Id: hal-00426813 https://hal.science/hal-00426813}

Submitted on 31 Jan 2017

HAL is a multi-disciplinary open access archive for the deposit and dissemination of scientific research documents, whether they are published or not. The documents may come from teaching and research institutions in France or abroad, or from public or private research centers.
L'archive ouverte pluridisciplinaire HAL, est destinée au dépôt et à la diffusion de documents scientifiques de niveau recherche, publiés ou non, émanant des établissements d'enseignement et de recherche français ou étrangers, des laboratoires publics ou privés. 


\title{
Soap Films Burst Like Flapping Flags
}

\author{
Henri Lhuissier and Emmanuel Villermaux* \\ Aix-Marseille Université, IRPHE, 13384 Marseille Cedex 13, France
}

(Received 4 February 2009; published 28 July 2009)

\begin{abstract}
When punctured, a flat soap film bursts by opening a hole driven by liquid surface tension. The hole rim does not, however, remain smooth but soon develops indentations at the tip of which ligaments form, ultimately breaking and leaving the initially connex film into a mist of disjointed drops. We report on original observations showing that these indentations result from a flaglike instability between the film and the surrounding atmosphere inducing an oscillatory motion out of its plane. Just like a flag edge flaps in the wind, the film is successively accelerated on both sides perpendicularly to its plane, inducing film thickness modulations and centrifuging liquid ligaments that finally pinch off to form the observed spray. This effect exemplifies how the dynamics of fragile objects such as thin liquid films is sensitive to their embedding medium.
\end{abstract}

DOI: 10.1103/PhysRevLett.103.054501

The speed at which a hole opens in a punctured soap film has been the subject of an interesting debate starting with Dupré (1868) [1] and Rayleigh (1891) [2] and ending with Taylor (1959) [3] and Culick (1960) [4]: Unbalanced surface tension forces at the hole edge set into motion the interstitial liquid within the film, and the constant receding velocity results from mass and momentum balances. However, most of the early contemplators of soap films $[5,6]$ paid little attention to the fragmentation of the film itself, which resolves into a mist of fine droplets which one can see as they remain suspended in the air after bursting. The fact that the film rim does not remain smooth as it recedes but becomes indented was already visible in pictures by Rayleigh [2] and Ranz [7], but it is McEntee and Mysels [8] who really identified these "fingers" as the objects responsible for the ejection of drops from the rim.

When an initially quiet soap film is punctured, the equilibrium of surface tension forces in the plane of the film is broken. Soap films are formed by pulling a rigid frame out of a solution of commercial detergent "Dreft" by Procter \& Gamble into tap water at a concentration above the critical micellar concentration with $\sigma_{0} \simeq$ $0.032 \mathrm{~N} \mathrm{~m}^{-1}$, and addition of disodium fluorecein when liquid fluorescence is needed. Bursting is performed into a vapor saturated atmosphere, recorded by a Photron digital camera with framing rate between 12500 and $25000 \mathrm{~s}^{-1}$. Film thicknesses $h_{0}$ at bursting range from 1 to $10 \mu \mathrm{m}$. If the initial hole diameter is typically larger than the film thickness, the hole opens, collecting the liquid constitutive of the film into a growing rim at its border, as seen in Fig. 1. For a film of thickness $h_{0}$, surface tension $\sigma_{0}$, and density $\rho$, the receding velocity is known as the Taylor-Culick velocity [3,4] $V=\sqrt{2 \sigma_{0} / \rho h_{0}}$. This sketch, which assumes a compact rim whose mass is concentrated at the edge, is relevant to pure water films [9-11] and predicts an accurate receding velocity for both pure water films [3] and soap films [8], the effect of the environment drag being sensitive
PACS numbers: 47.55.nm, 47.20.Dr, 47.20.Ft, 47.20.Ma

for very thin films $\left(h_{0}<0.1 \mu \mathrm{m}\right)$ only $[12,13]$. When observed on a longer time period, the rim is seen to indent spontaneously, a phenomenon which has been thought to result from some instability intrinsic to the recession kinetics itself $[8,14]$. However, if the phenomenon is contemplated from a different perspective than the traditional frontal point of view, namely, if one looks at an angle, one discovers the existence of motions of the film perpendicular to its plane in an oscillatory, flappinglike fashion. The flapping motion is obvious in the detailed lateral view of Fig. 1 and in Fig. 2. The striking similarity of Fig. 2 with flags and sails flapping in the wind strongly suggests that an interaction with the surrounding atmosphere has to be considered at the root of the phenomenon [15]. The similarity with the flapping of rapidly moving liquid sheets in a quiet gaseous phase, familiar in the liquid atomization context [16-18], is even stronger.

The origin of the soap film flapping is indeed a shear instability with the outer medium and results from the velocity difference between the moving film and the atmosphere at rest. The tangential velocity difference between both phases amplifies crest and trough amplitudes on both liquid-gas interfaces of the film. This coupling, of a Kelvin-Helmholtz type [16], allows for two distinct modes: a varicose one, in which both interfaces are in phase opposition and the film thickness modulates, and a sinuous one, in which interfaces are in phase and oscillate the film at constant thickness-this is the flapping mode. For all wavelengths, the flapping mode is always dominant $[16,19]$. Its most amplified wavelength $\lambda$ and pulsation $\omega$ can be inferred by assuming, for simplicity, an infinite, uniform film of thickness $h_{0}$ in relative motion at velocity $V$ with an outer environment of density $\rho_{a}$ (the relevance of these hypotheses is discussed below)

$$
\lambda \sim \frac{\sigma_{0}}{\rho_{a} V^{2}} \quad \text { and } \quad \omega \sim \frac{V}{\lambda} \sim \frac{\rho_{a} V^{3}}{\sigma_{0}} .
$$



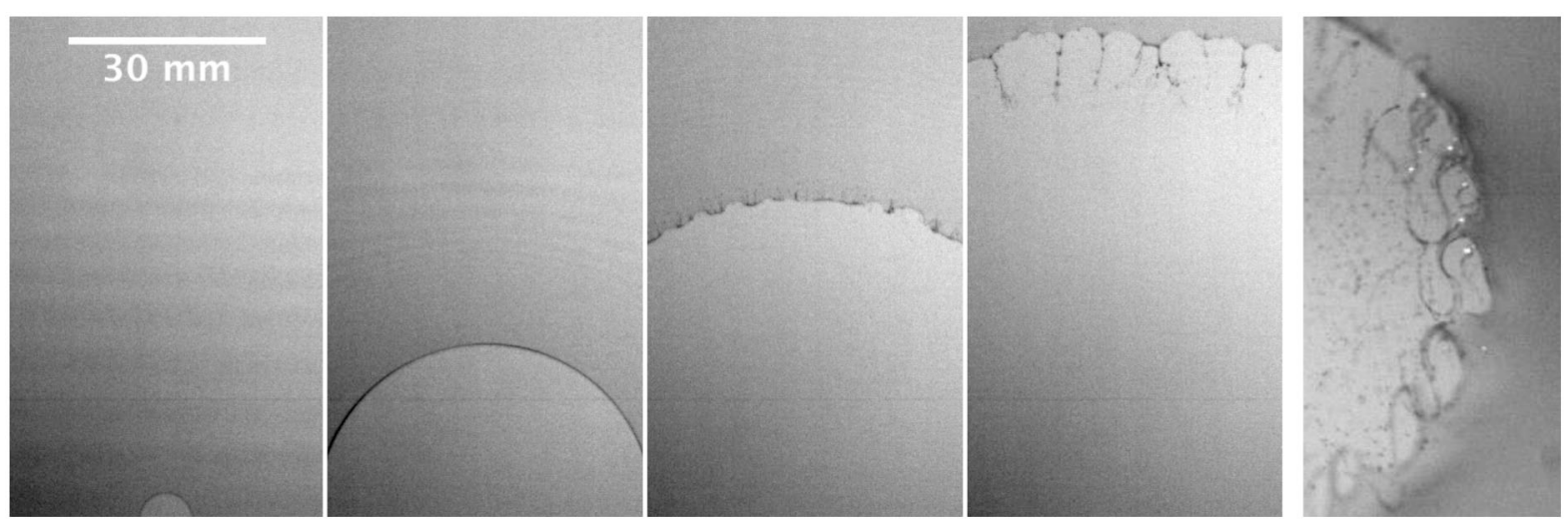

FIG. 1. Destabilization of a soap film receding edge. Left: Sequence of the opening of a soap film punctured by the projection of a spark. In the two first images, the film edge is smooth. Growing corrugations appear in the third image and reach a saturated size. Indentations (or fingers) obvious in the last image are ligaments draining the liquid and continually pinching off to form the resulting mist. Images are sampled every $6.7 \mathrm{~ms}$. Right: Snapshot emphasizing on indentations. Note the arabesques, ligaments detaching from the rim as they flap like a whip.

Figure 2 shows experimental measurements of the flapping pulsation in ambient air. Both the trend and the absolute values follow the anticipated scaling law in Eq. (1), giving support to the shear instability scenario. A further confirmation of the role played by the outer medium is provided by the study of the delay time for the onset of rim indentations, that we conducted into two distinct atmospheres. We bursted soap films not only in air but also in sulfur hexafluoride $\left(\mathrm{SF}_{6}\right)$, a gas 5 times denser than air at atmospheric pressure, and we measured the delay time $\tau_{d}$ for the indentations depicted in Fig. 1 to appear. Figure 3 shows a plot of this delay time $\tau_{d}$ against the receding velocity $V$ for both atmospheres. A fact immediately obvious is that the rim destabilization develops earlier in a denser atmosphere, everything else being identical since to one receding velocity $V$ is associated a unique film thickness $h_{0}$. A precise interpretation of the dependence of $\tau_{d}$ on $\rho_{a}$ requires understanding how those indentations form. We suggest an analogy with the "wavy corridor" mechanism proposed in the context of liquid sheets fragmentation $[19,20]$.

The wavy pattern consecutive of the flapping instability propagates at a group velocity proportional to $V$ on the film. A fluid particle in the flapping region thus experiences transient accelerations perpendicular to the film plane (whose intensity is $\gamma=a \omega^{2}$ for a flapping amplitude $a$ ) as the wave propagates. This, in turn, initiates a RayleighTaylor instability of the film which causes film thickness modulations at a transverse wavelength $\lambda_{\perp}$, precursors of the rim indentations visible in Fig. 1. The full mechanism is thus a succession of two destabilizations of the film. The first shear instability breaks the symmetry with respect to the film median plane, and a second inertial instability of the undulating substrate breaks the azimuthal invariance by forming indentations. This last destabilization is not instantaneous, as Fig. 1 clearly shows. The rim remains smooth during an initial transient (lasting $\tau_{d}$ ), and it then suddenly corrugates to form the fingers from which droplets are ejected. Flapping is, however, present almost since the beginning of the hole opening, but its amplitude $a$ is not strong enough to trigger thickness modulations within the acceleration permanency time, of the order of $\omega^{-1}$. The delay $\tau_{d}$ is scaling like the flapping instability growth time $\tau$, itself given by $\omega^{-1}$, as it is standard for all shear instabilities whose growth is controlled by the linear development stage before saturation. Assuming a plausible initial noise amplitude on the film of $a_{0} \sim 10^{-8} \mathrm{~m}$ for a wavelength $\lambda \sim 1 \mathrm{~mm}$, the delay time required to reach the rupture amplitude $a \sim 10^{-3} \mathrm{~m}$ suggested by Fig. 2 is close to $5 \ln (10) \tau$. During this growth period, an unstable shear wave must have been traveling (with celerity of order $V$ ) within the unstable interval of the aureole, whose width is about $V t / 4$ [21]. Thus, $V \times 5 \ln (10) \tau=V \tau_{d} / 4$, that is, $\tau_{d} \simeq 20 \ln (10) \tau \simeq 50 \omega^{-1}$. As seen in Fig. 3, a good collapse is obtained for both atmospheres according to the expected trend when $\rho_{a}$ has varied by a factor of 5, with a prefactor of the order of 50 between $\tau_{d}$ and $\omega^{-1}$.

Once the nature of the rim destabilization is understood, a prediction on its azimuthal wavelength $\lambda_{\perp}$ can be made. The transverse accelerations $\gamma$ perpendicular to the film and associated capillary wave number $k_{\perp c}=\sqrt{\rho \gamma / \sigma}$ are such that $h_{0} k_{\perp c} \ll 1$ (Figs. 1 and 4). In that case, the modified Rayleigh-Taylor instability accounting for the finite film thickness [22] leads to a most amplified wavelength $\lambda_{\perp}$ and growth rate $\tau_{\perp}^{-1}$ given by

$$
\frac{1}{\tau_{\perp}}=\sqrt{\frac{\gamma}{\lambda_{\perp}}} \sim \sqrt{\frac{\rho \gamma^{2} h_{0}}{\sigma_{0}}} \text { and } \lambda_{\perp} \sim\left(k_{\perp c}^{2} h_{0}\right)^{-1} .
$$

The onset for the secondary wavy corridor mechanism occurs when $\tau_{\perp} \sim \omega^{-1}$, expressing that its growth time must match the permanence time of the acceleration imposed by the flapping motion [19], giving 


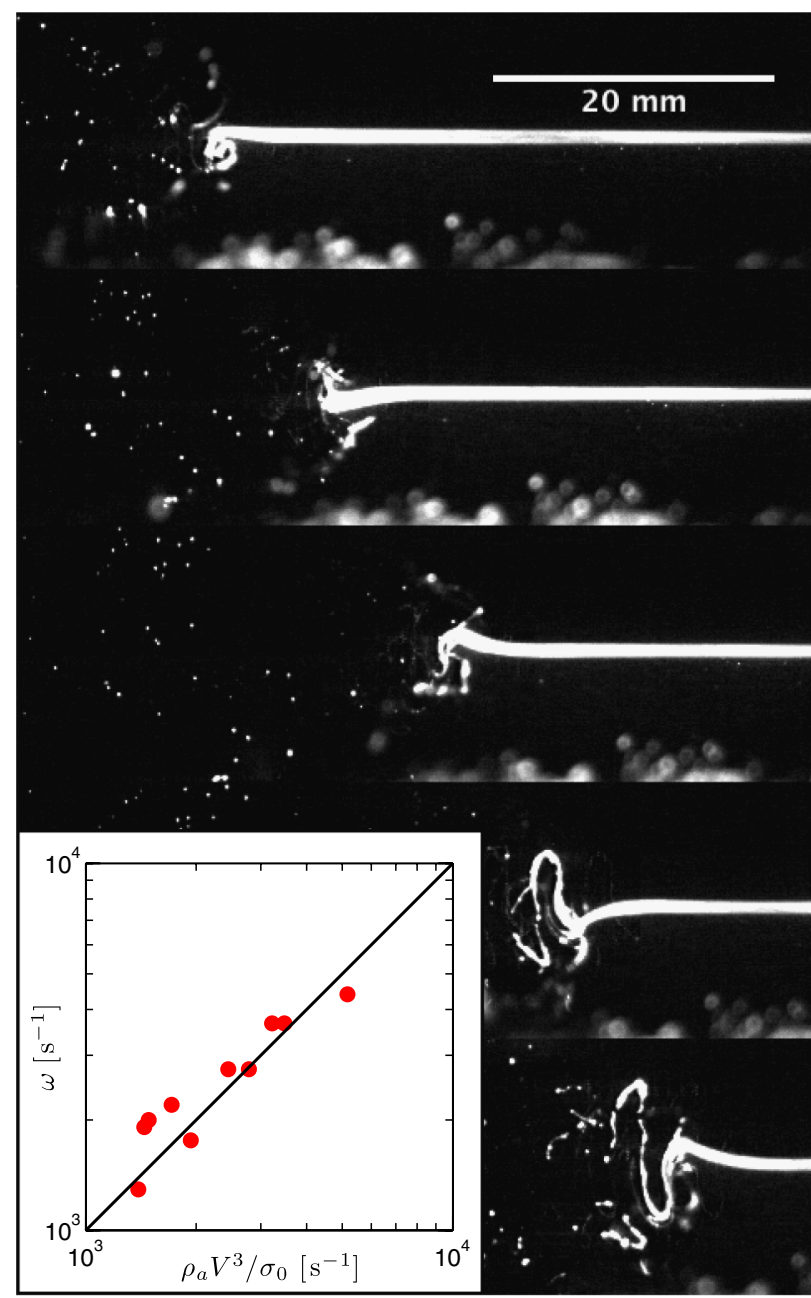

FIG. 2 (color online). Flapping motion. Main: Sequence of a flapping receding soap film from a lateral view. A laser sheet under normal incidence lights the fluorescent film emphasizing the out of plane motions. The initially flat film is punctured on the left-hand side and, as it recedes, initiates a characteristic flapping motion close to its edge which grows in amplitude. Images are sampled every $2.3 \mathrm{~ms}$. Inset: Flapping pulsation $\omega$ versus $\rho_{a} V^{3} / \sigma_{0}$, where $\rho_{a}$ is the outer medium density. The solid line is the expected scaling law in Eq. (1).

$$
\lambda_{\perp} \sim \sqrt{\frac{\sigma_{0} \tau_{\perp}^{2}}{\rho h_{0}}}=\frac{V}{\omega}=\lambda .
$$

The experimental measurements of $\lambda_{\perp}$ reported in Fig. 4 are, within some scatter, consistent with this trend.

The success of the vision that we have proposed to explain both film flapping and consecutive indentations must not hide the subtle discussion which legitimates its use in practice. By contrast with the classical KelvinHelmholtz waves developing at the interface between two infinite media with velocity difference $u$, the case of a film with finite thickness $h$ moving at speed $u$ and sandwiched between two infinite media presents a threshold above which destabilization can occur. That threshold

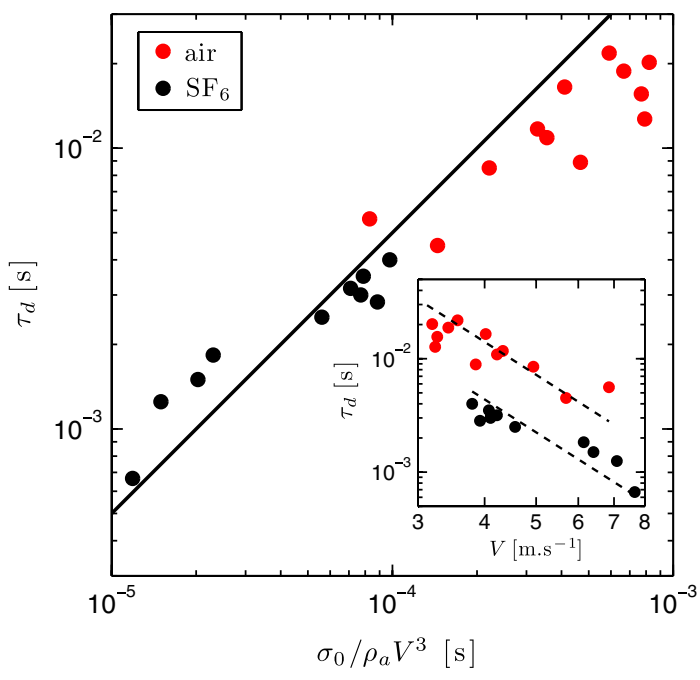

FIG. 3 (color online). Influence of the outer medium density. Inset: Delay time $\tau_{d}$ before rim destabilization versus receding velocity $V$ for films bursting in air and in sulfur hexafluoride $\left(\mathrm{SF}_{6}\right)$. The dotted lines have a slope -3 . Main: Delay $\tau_{d}$ versus $\sigma_{0} / \rho_{a} V^{3}$; the solid line is the expected scaling law.

expresses $[16,19]$ that the Weber number

$$
\mathrm{We}=\frac{\rho h u^{2}}{\sigma}
$$

should be larger than 2. However, the sketch of a concentrated rim with $u=V$ and $\sigma=\sigma_{0}$ predicts exactly a marginally critical state of the film with respect to the flapping instability since $\mathrm{We}=2$ for that motion. As it is, the concentrated rim idealization thus fails at explaining the flapping destabilization of the film edge, nevertheless obvious in, e.g., Fig. 2. A more detailed study of the rim

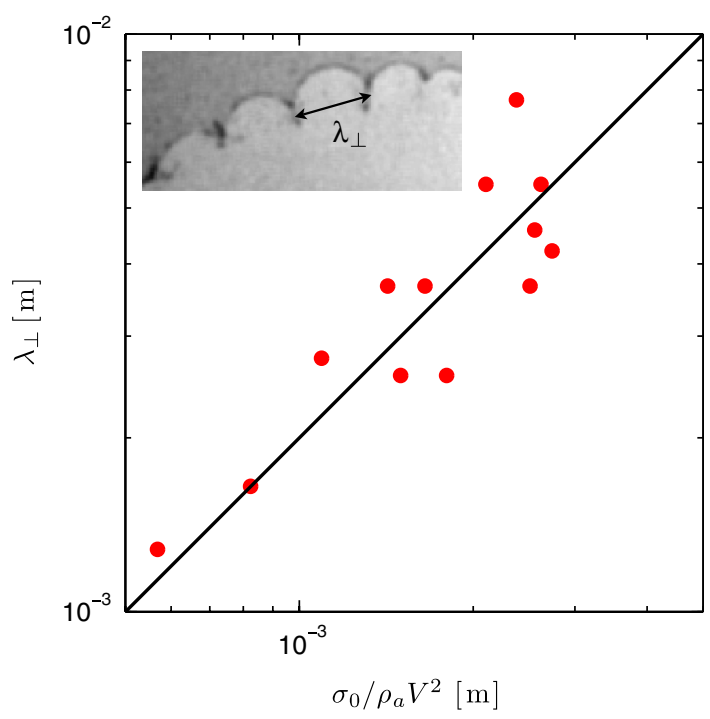

FIG. 4 (color online). Indentation size. Indentation transverse wavelength $\lambda_{\perp}$ as defined in the inset versus $\sigma_{0} / \rho_{a} V^{2}$. The solid line is the expected scaling law. 
shape and its interstitial flow field solves nevertheless this paradox.

A precise characterization of the flow ahead from an opening hole in a soap film was first made by Frankel and Mysels [23], motivated by the discovery by McEntee and Mysels [8], using interferometric methods, of an "aureole" surrounding the hole. This extended rim corresponds to a region preceding the film edge where the film thickens. Its origin lies in the special properties of surfactants at the interface. Surfactants, in addition to globally decreasing surface tension of an interface at equilibrium, can cause local variations in surface tension if they are not evenly concentrated at the surface and may thus give birth to large scale flows. When a soap film recedes, its interfacial area rapidly shrinks and its surfactant surface concentration locally increases. This, in turn, decreases locally surface tension and creates a surface tension gradient which sets the fluid into motion in what is referred to as a Marangoni flow. Those local variations in surface tension are likely to occur only because deformations are fast enough compared to surfactant molecule equilibration time with the liquid bulk $[24,25]$. They induce the spreading of an aureole where the film thickens and the fluid is gradually set into motion, far ahead from the film edge up to the unperturbed portion of the film. Its radial extension grows with time, scaling at any instant like the hole diameter.

A simple model can be built assuming that surfactant molecules at the interface are passively convected by the inner flow. Surface tension is thus locally linked to the interfacial area shrinking and then via mass conservation to the local film thickness. Frankel and Mysels [23] solved the corresponding system of continuity and momentum equations leading, with no surprise, to fluid velocity $u(r, t)$ and film thickness $h(r, t)$ fields (where $r$ is the radial coordinate) which are self-similar functions of $r /(V t)$. Experimental evidence for these aureoles confirming their quantitative properties was later provided $[14,21,26]$. The major conclusion is that, in the vicinity of the hole in the extended rim, the film velocity is $u \sim V$, the local thickness $h$ is larger than the initial thickness $h_{0}$, and the local surface tension $\sigma$ is smaller than the initial surface tension $\sigma_{0}$ since film thickening implies surface shrinking and therefore surface tension decrease. This all together leads to a local Weber number We given in Eq. (4) larger than 2 over a broad region whose extension is typically a quarter of the current hole radius, which becomes rapidly larger than $\lambda$ : This is the region that experiences flapping. Yet, flapping results from an interaction with the outer medium and is not an intrinsic oscillation due to the continuously decreasing tension along the aureole, as for flexible beams hanging in gravity [27].

The flapping phenomenon documented here is inherent to soap film recession. Every liquid film containing surfac- tants and bursting in a nonvacuum atmosphere will flap and atomize into droplets under its own recession motion. The only condition is that the film extension has to be larger than the typical length over which flapping develops: $\lambda \sim$ $\left(\rho / \rho_{a}\right) h_{0}$. The indentation phenomenon will be hastened if a curvature preexists on the film, such as for bubbles bursting spontaneously. In that case, the acceleration responsible for the rim destabilization will be set by the initial bubble radius of curvature, a crucial ingredient accounting for the production of aerosols at the sea surface.

*Also at Institut Universitaire de France, Paris, France. villermaux@irphe.univ-mrs.fr

[1] A. Dupré, Ann. Chim. Phys. 11, 194 (1867).

[2] L. Rayleigh, Nature (London) 44, 249 (1891).

[3] G. I. Taylor, Proc. R. Soc. A 253, 313 (1959).

[4] F. E. C. Culick, J. Appl. Phys. 31, 1128 (1960).

[5] H. Bouasse, Capillarité (Delagrave, Paris, 1924).

[6] C. V. Boys, Soap-Bubbles (Dover, New York, 1959).

[7] W.E. Ranz, J. Appl. Phys. 30, 1950 (1959).

[8] W. R. McEntee and K. Mysels, J. Phys. Chem. 73, 3018 (1969).

[9] J. B. Keller and M. Miksis, SIAM J. Appl. Math. 43, 268 (1983).

[10] M. Brenner and D. Gueyffier, Phys. Fluids 11, 737 (1999).

[11] G. Sünderhauf, H. Raszillier, and F. Durst, Phys. Fluids 14, 198 (2001).

[12] K. Mysels and B. R. Vilayendran, J. Phys. Chem. 77, 1692 (1973).

[13] G. Frens, J. Phys. Chem. 78, 1949 (1974).

[14] N. Y. Liang, C. K. Chan, and H. J. Choi, Phys. Rev. E 54, R3117 (1996).

[15] L. Rayleigh, Proc. London Math. Soc. s1-10, 4 (1878).

[16] H. B. Squire, Br. J. Appl. Phys. 4, 167 (1953).

[17] J. Lucassen, M. V. D. Tempel, A. Vrij, and F. T. Hesselink, J. Phys. Chem. B 73, 109 (1970).

[18] E. Villermaux, Annu. Rev. Fluid Mech. 39, 419 (2007).

[19] E. Villermaux and C. Clanet, J. Fluid Mech. 462, 341 (2002).

[20] N. Bremond, C. Clanet, and E. Villermaux, J. Fluid Mech. 585, 421 (2007).

[21] H. Lhuissier and E. Villermaux (to be published).

[22] N. Bremond and E. Villermaux, J. Fluid Mech. 524, 121 (2005).

[23] S. Frankel and K. Mysels, J. Phys. Chem. 73, 3028 (1969).

[24] P. F. Mijnlieff and R. Ditmarsch, Nature (London) 208, 889 (1965).

[25] A. Rusanov and V. Krotov, Prog. Surf. Sci. 13, 415 (1979).

[26] A. T. Florence and G. Frens, J. Phys. Chem. 76, 3024 (1972).

[27] D. Bernoulli, Comment. Acad. Scient. Imp. Petropolit. 6, 108 (1738). 